\title{
Soft Robotics for Chemists
}

\section{Citation}

Ilievski, Filip, Aaron D. Mazzeo, Robert F. Shepherd, Xin Chen, and George M. Whitesides. 2011.

"Soft Robotics for Chemists." Angewandte Chemie International Edition 50, no. 8: 1890-1895.

\section{Published Version}

doi:10.1002/ange.201006464

\section{Permanent link}

http://nrs.harvard.edu/urn-3:HUL.InstRepos:12967812

\section{Terms of Use}

This article was downloaded from Harvard University's DASH repository, and is made available under the terms and conditions applicable to Open Access Policy Articles, as set forth at http:// nrs.harvard.edu/urn-3:HUL.InstRepos:dash.current.terms-of-use\#OAP

\section{Share Your Story}

The Harvard community has made this article openly available.

Please share how this access benefits you. Submit a story.

Accessibility 


\section{Soft Robotics for Chemists}

Filip Ilievski ${ }^{1}$, Aaron Mazzeo ${ }^{1}$, Robert F. Shepherd ${ }^{1}$, Xin Chen ${ }^{1}$, and George M. Whitesides ${ }^{1,2, *}$

${ }^{1}$ Department of Chemistry and Chemical Biology, Harvard University 12 Oxford Street, Cambridge, MA 02138

${ }^{2}$ Kavli Institute for Bionano Science \& Technology, 29 Oxford Street, Cambrdige MA, and Wyss Institute for Biologically Inspired Engineering, Harvard University 3 Blackfan Circle, Boston, MA 02115

* corresponding author: gwhitesides@gmwgroup.harvard.edu 


\begin{abstract}
The development of new types of soft robots, and especially of materials and methods for the fabrication of this class of robots, requires and offers rich new opportunities for interdisciplinary research involving organic chemistry, soft materials science, and robotics. This paper describes a methodology based on embedded pneumatic networks (PneuNets) that enables large-amplitude actuations in soft elastomers by pressurizing embedded channels. Examples include a structure that can change its curvature from convex to concave, and two devices that act as compliant grippers for handling fragile objects (e.g., an uncooked chicken egg, a live, anesthetized mouse) without damaging either. These systems suggest new areas of research for organic materials that are relevant to soft robotics, and to which chemists can make important contributions.
\end{abstract}
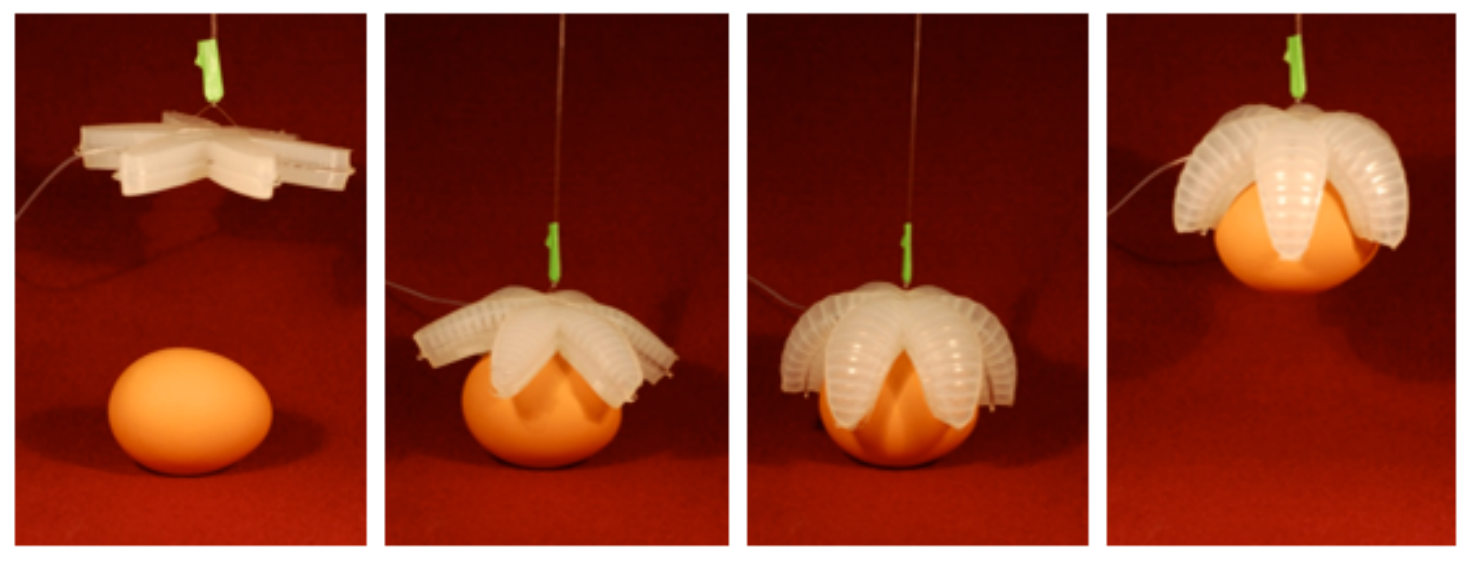
In areas from assembly of machines ${ }^{[1]}$ to surgery ${ }^{[2]}$, and from deactivation of improvised explosive devices (IEDs) to unmanned flight, robotics is an important and rapidly growing field of science and technology. It is currently dominated by robots having hard body plans - constructions largely of metal structural elements and conventional joints $^{[3]}$ - and actuated by electrical motors, or pneumatic or hydraulic systems. Handling fragile objects - from the ordinary (fruit) to the important (internal organs) - is a frequent task whose importance is often overlooked and is difficult for conventional hard robots; moving across unknown, irregular, and shifting terrain is also. Soft robots may provide solutions to both of these classes of problems, and to others. Methods of designing and fabricating soft robots are, however, much less developed than those for hard robots. We wish to expand the methods and materials of chemistry and soft-materials science into applications in fully soft robots.

A robot is an automatically controlled, programmable machine ${ }^{[4]}$. The limbs of animals or insects - structures typically based on rigid segments connected by joints with constrained ranges of motion ${ }^{[5]}$ - often serve as models for mobile elements of robots. Although mobile hard robots sometimes have limb-like structures similar to those of animals (an example is "Big Dog" by Boston Robotics ${ }^{[6]}$ ), more often, robots use structures not found in organisms - for example, wheels and treads.

The robotics community defines "soft robots" as: i) machines made of soft often elastomeric - materials, or ii) machines composed of multiple hard-robotic actuators that operate in concert, and demonstrate soft-robot-like properties ${ }^{[7]}$; here, we consider only the former. Soft animals offer new models for manipulation and mobility not found, or generated only with difficulty and expense, using hard robots. Because 
materials from which this class of devices will be fabricated will usually be polymers (especially elastomers), they fall into the realm of organic materials science. The use of soft materials allows for continuous deformation; this type of deformation, in turn, enables structures with ranges of motion limited only by the properties of the materials.

Soft robots have the potential to exploit types of structures found, for example, in marine organisms ${ }^{[8]}$, and in non-skeletal parts of land animals ${ }^{[9]}$. The tentacles of squid, trunks of elephants, and tongues of lizards and mammals are such examples; their structures are muscular hydrostats ${ }^{[10][11]}$. Squid $^{[12]}$ and starfish ${ }^{[13][14]}$ are highly adept locomotors; their modes of movement have not been productively used, and permit alternative solutions of manipulation, locomotion, and navigation, to those used in conventional hard robotics.

The prototypical soft actuator - muscle - developed through the course of evolution. There is currently no technology that can replicate the balanced performance of muscle ${ }^{[15]}:$ it is simultaneously strong and fast, and enables a remarkable range of movements (such as those of a tongue). Muscle-like contraction and dilation occur in ionic polymeric gels on changes in the acidity or salinity of a surrounding ionic solution, but actuation in macroscopic structures is mass-transport limited, and typically slow ${ }^{[16]}$. Other electroactive polymers (EAPs) include dielectric elastomers, electrolytically active polymers $^{[17]}$, polyelectrolyte gels ${ }^{[18]}$, and gel-metal composites ${ }^{[16]}$.

Pneumatically-driven McKibben-type actuators are among the most highly developed soft actuators, and have existed for more than fifty years; they consist of a bladder covered in a shell of braided, strong, inextensible fibers ${ }^{[19]}$. These actuators can be fast, and have a length-load dependence similar to that of muscle ${ }^{[19]}$ but possess only 
one actuation mode - contraction and extension when pressurization changes. They are, in a sense, an analogue to a single muscle fibril; using them for complex movements requires multiple actuators acting in series or parallel. Pneumatically-driven flexible microactuators (FMAs) have been shown to be capable of bending, gripping, and manipulating objects ${ }^{[20-23]}$. Roboticists have explored scalable methods for gripping and manipulating objects at the micro and nano scales ${ }^{[24-27]}$. The use of compliant materials allows grippers to manipulate objects such as fruit ${ }^{[28]}$ with varied geometry ${ }^{[29]}$.

The field of robotics has not yet caught the attention of soft-materials scientists and chemists. Developing new materials, techniques for fabrication, and principles of design will create new types of soft robots.

The objective of this work is to demonstrate a type of design that provides a range of behaviors, and that offers chemists a test bed for new materials and methods of fabrication for soft robots. Our designs use embedded pneumatic networks (PneuNets) of channels in elastomers that inflate like balloons for actuation ${ }^{[30]}$. We used a series of parallel chambers embedded in elastomers as repeating components. Using intuition and empirical experimentation, we stacked ${ }^{[31]}$ or connected these repetitive components to design and test prototypical structures that provide complex motion. In this type of design, complex motion requires only a single source of pressure; appropriate distribution, configuration, and size of the PneuNets determine the resulting movement.

We selected pneumatic systems as a strategy for supplying energy (compressed air at 7 to $28 \mathrm{kPa}$ or $1-4 \mathrm{psig}$ ). Air has low viscosity, and permits rapid actuation; since air is compressible, it is easy to store, light and environmentally benign. When pressurized, the channels expand in the regions that are most compliant or have the lowest stiffness 
(Figure 1). In homogeneous elastomers, such regions are those defined by the thinnest walls (i.e., by the structure with the lowest resistance to stretching). Pressurization and expansion in these regions further thins the walls, and increases the volume of the channel. To accommodate the asymmetric elongation of two opposite walls of the channel, the structure surrounding the expanding volume bends. Upon pressurization, a single channel spanning the length of a rectangular slab causes the slab to bend around the axis of the channel. Multiple channels have an additive effect; upon pressurization, an actuated network of channels can generate complex shapes in elastomeric structures.

Describing the expansion of thin-walled balloons analytically is a well understood problem, but the complex structure used here, where different walls have different thicknesses, and the inflation is constrained on one dimension, does not have a simple analytical model; we can, however, use finite element analysis to model expanding structures $^{[32][33]}$ (Figure 1 b).

The choice of materials, coupled with the design of the channels, determines the response of the device to applied pressure. The pressure necessary to achieve a particular amplitude of actuation scales with the stiffness of the materials. Figure $2 \mathrm{~b}$ compares the performance of PneuNets fabricated from different materials. When pressurized to $14 \mathrm{kPa}$ ( 2 psig), the rectangular slabs in the stiffer material had a minimum radius of curvature of $\sim 15 \mathrm{~cm}$; the same structure made from a softer, more elastic material, had a radius of curvature of approximately $1.5 \mathrm{~cm}$. Passive and active loading capacity of actuators (the maximum sustainable load) correlates to the stiffness of the materials used for fabrication - stronger materials are able to support larger loads, but typically cannot sustain very large strains. Agility - the ability to create intricate movements, and to do so rapidly - 
requires bending to small radii of curvature, and is thus easier to achieve in materials able to sustain high strains.

Composite structures, in which materials with different stiffnesses join to form a channel, are useful for programming the directionality of actuation, and provide properties that benefit from the combined mechanical properties of the different materials. As with single-material devices, the composite channels expand upon pressurization at the most compliant region; since the different compliances are now not controlled by the channel design, but largely determined by the choice of materials and their layout, the region made from a softer, more elastic material will expand (compare different designs in Figure $2 \mathrm{c}$ ).

We used two silicone elastomers (polydimethylsiloxane (PDMS, Dow Corning Sylgard 184) and Ecoflex 00-30 (a siloxane produced by Smooth-On; http://www.smooth-on.com)) because they are easily accessible, are easy to work with, bond well to each other to form multilayer structures, and are relatively inexpensive. PDMS is transparent and has a Shore A hardness of $50^{[34]}$. It is elastic and can withstand repeated bending, but fractures above a maximum strain of $150 \% \cdot{ }^{[34]}$ As a result, PDMS has a limited range of deformation, and is best suited for the more rigid parts of a structure - parts that bend but do not stretch. Ecoflex is translucent and has a hardness below the Shore A scale ${ }^{[34]}$. It fractures only above a maximum strain of $900 \%{ }^{[34]}$; it is more flexible than PDMS, and therefore, it is suitable for components with larger strains/displacements (i.e., the layers of actuation). Because it is so soft, Ecoflex, if unsupported, will bend under its own weight (PDMS will not). Composite structures, comprising layers of PDMS and Ecoflex, balance the rigidity of PDMS with the 
flexibility of Ecoflex for optimal function. Alternate materials are useful for the fabrication of devices - composites with paper, textiles, carbon-, glass- or metal fiber are possible: for example, Figure $2 \mathrm{c}$, right shows a device in which the strain-limiting layer is a $100-\mu \mathrm{m}$ thick polyester fabric encapsulated in Ecoflex.

Forming channels in silicones and other elastomers is a well understood, widely used technique in soft lithography and microfluidics ${ }^{[35]}$. In this paper, we have applied these techniques to the construction of soft robotic PneuNets. We prepared the molds using 3D printing ${ }^{[36][37]}$; we first designed the molds using computer-aided design (CAD; Alibre, Inc.) and generated them using a three-dimensional (3D) printer (StrataSys Dimension Elite) with acrylonitrile butadiene styrene (ABS) plastic; 3D printing is a rapid, low-cost process, and well suited for rapid prototyping (Error! Reference source not found.). We formed the channels by first casting a prepolymer in the mold, partially cured the molded prepolymer by a short (15 minute) baking step, and removed the resulting open-channel structure from the mold. To fabricate the final closed-channel devices, we bonded the partially cured, molded open-channel piece to a flat membrane by manually applying a thin coat of uncured prepolymer (of the same material as the membrane) to serve as an adhesive. We joined the parts and let the entire structure cure fully by baking it at $60^{\circ} \mathrm{C}$ for $3 \mathrm{~h}^{[38]}$.

A fully soft robotic gripper provides a demonstration of the class of soft structures that can be generated with these methods. This gripper incorporates a starfish-like structure with pneumatically-driven soft actuators fabricated in elastomeric composites. When compressed air pressurizes the PneuNet of channels, the soft actuators bend, extend, or contract to a pre-programmed shape. 
PneuNets can generate a wide (if not universal) variety of movements. We have fabricated elements ("fingers") which curl around an axis perpendicular to the length of the device. Slabs that coil in a helix use channels that are at an angle with respect to the long axis of the finger; the tilting angle of the channels (positive or negative) determines the handedness of the helical structure (Error! Reference source not found.). Combination of multiple elements in a single structure forms networks capable of complex movement. For example, by placing six of these elements radially around a common inlet, we formed the starfish-like structure shown in Figure 4.

Multilayer structures, where layers of actuation sandwich passive layers, can form devices with a wide range of motions. A three-layer starfish-like structure consisting of two active layers separated by a single flat membrane provides an example (Figure $4 \mathrm{a}$ and b). Each of the top and bottom Ecoflex-based layers contained one set of embedded channels, which ran almost the entire lengths of the legs. Between these two molded layers, a flat PDMS membrane served to seal both sets of channels. By actuating the individual networks, the device could continuously change its shape from convex to concave (Figure 4b). The freedom to design each layer separately, and then join them in a single device, made it possible to tailor the structure of each part to achieve a function. For example, a ridged texture added to a layer enhanced gripping ${ }^{[39]}$ (Figure 5). This textured surface is more compliant than a solid surface of the same material and provided more traction; other physical or chemical modifications to the gripping surface could also be applied ${ }^{[40]}$.

To demonstrate a soft structure with immediate potential for use, we built starfishlike grippers. The active parts of these structures are fully soft; PDMS and Ecoflex are 
the only materials used for fabrication; the polyethylene tubing and nylon string used for lifting are not. The tri-layer structure used here consisted of one active layer made of Ecoflex, a closing PDMS membrane and a gripping layer formed of Ecoflex. The gripping layer was either a solid layer of Ecoflex or a textured surface shown in Figure 5. Each of the networks for actuating the individual legs used a common inlet; polyethylene tubing inserted in the inlet delivered air for actuation. The actuated legs of the gripper curled about axes perpendicular to the long axes of the legs. When we applied pressure to the PneuNets in the top active layer of the device, the legs curled downward; the actuated response by the effective curling of the six legs was capable of gripping objects such as an uncooked egg and an anesthetized mouse (Figure 6). The soft gripper did not damage/harm the egg or the mouse during manipulation.

By picking up smooth polypropylene spheres with increasing diameter and weight, we tested the loading capacity of the grippers; we used spheres with diameters of $2.5,5,7.5$, and $10 \mathrm{~cm}$ and incrementally loaded them with free weights until the gripper failed to lift and maintain a grip on the sphere. This test showed that the grippers described here were able to maintain a grip on spheres with diameters up to $10 \mathrm{~cm}$ and loads up to 300 grams. Adding a modified surface such as that depicted in Figure 5 to both grippers improved their ability to lift larger and heavier spheres. The supplemental information includes tabulated values for the maximum loads attained.

Soft robots are promising for applications that require robotic interactions with delicate objects, and where the use of feedback sensors is too cumbersome, difficult, or costly to install. The flexible pneumatic manipulators, upon gripping, do not concentrate stresses in the way that rigid manipulators do - the soft pneumatically actuated interface 
distributes the force over the entire area of contact. Patterning topography into the gripping surface created alternating gaps of air and Ecoflex, increased the compliance of the surface and improved their ability to grip fragile objects. Because the PneuNets do not use articulated joints the soft grippers can manipulate many irregularly shaped objects. Even when actuated by a single inlet, fingers with PneuNets can have different local curvatures along their length. Therefore, edges or protrusions on an object do not interfere with the actuation or gripping, and the compliant gripping surface can closely follow an irregular surface. Distributed pneumatic networks also increase the flexibility of actuation: if a part of the network is prevented from expanding (actuating), the remaining part of the network will still be able to function properly. PneuNets do not require high power to deliver versatile function. For the devices described here, we estimate a consumption of about $4 \mathrm{~W}$ per stroke. Continuous feeding of power to maintain an actuated (or gripped) state is not necessary: when a simple valve is closed, the device (gripper) remains in the inflated/closed state. Rates of gas permeability through the elastomers and leaks at inlets limit the duration, but our un-optimized prototypes were able to maintain the same shape for 10 minutes without significant changes in shape, and required 60 minutes for full deflation.

The soft materials described here are not suitable for manipulating heavy objects. The methods, however, are versatile and, with an appropriate choice of materials, could be extended to applications that require high loads. Hydraulic actuation - actuation using fluids rather than gas - are amenable to similar designs and are useful for situations that require greater force, or incompressible robotic modules, or density (of the robot) matched to that of an aqueous environment (for example, for applications underwater). 
Soft robotics offers many opportunities to organic materials science, especially in areas related to soft elastomers. The system of actuation described here - one based on pneumatic expansion in easily fabricated networks of small channels - will, we believe, provide a test bed for new materials - especially elastomers - and concepts of design for soft robots and robotic components. Development of elastomeric materials with high strength, which can also sustain large strains without fracture, would enable soft robots that are both agile and strong. Materials with variable compliance could be useful for agile machines that could locally change the properties of the material and enable fundamentally new strategies for manipulation. New adhesives would make it easier to bond layers. Soft robots with components that are electrically conductive, optically transparent, independently functional (e.g. as sensors, fluidic devices, transducers or analog computers) or biocompatible could open entirely new typesr of applications, but would require materials with appropriate properties. Anisotropic materials would provide the basis for a wide range of non-linear motions.

\section{Acknowledgments}

This work was supported by DARPA under award number W911NF-08-1-0143. We thank Dr. Joe Foley from iRobot and Dr. Cagdas Onal from MIT for initial help with 3D printing. 


\section{Bibliography}

[1] A. Quaid, R. Hollis, in Proc. IEEE Int. Conf. Rob. Autom., Minneapolis, MN, USA, 1996, pp. 2188-2193.

[2] F. Gharagozloo, F. Najam, Robotic Surgery, McGraw-Hill Medical, New York, 2009.

[3] J. A. Angelo, Robotics: A Reference Guide to the New Technology, Greenwood Press, Westport, Connecticut, 2007.

[4] ISO - International Organization for Standardization, ISO 8373:1994, Manipulating Industrial Robots, Vocabulary; can be found under http://www.iso.org/iso/catalogue_detail.htm?csnumber=15532.

[5] An alternative description is to use the kinematics concept of degree of freedom (DOF), described as "the number of parameters needed to specify the configuration of a mechanism, in terms of the number of links and joints and the freedom of movement allowed at each joint. This number is the degree of freedom or mobility of the mechanism. Changing the values of these parameters changes the configuration of the mechanism." from J. M. McCarthy, Introduction to Theoretical Kinematics, The Mit Press, Cambridge, USA, 1990. A hard robot is said to have limited number of DOF.

[6] M. Raibert, K. Blankespoor, G. Nelson, R. Playter, BigDog Team, BigDog, the Rough-Terrain Quadruped Robot, Boston Dynamics, 2008; can be found under http://www.bostondynamics.com/img/BigDog_IFAC_Apr-8-2008.pdf. 
[7] A. Crespi, A. Badertscher, A. Guignard, A. J. Ijspeert, Robot. Auton. Syst. 2005, 50, $163-175$.

[8] S. Vogel, Life in Moving Fluids: The Physical Biology of Flow, W. Grant Press, Boston, Mass., 1981.

[9] S. Vogel, Prime Mover: A Natural History of Muscle, W.w. Norton, New York, 2001.

[10] D. Trivedi, Appl. Bionics and Biomech. 2008, 5, 99-117.

[11] W. Kier, K. Smith, Zool. J. Linn. Soc. Lond. 1985, 83, 307-324.

[12] J. Wilson, D. Li, Z. Chen, R. George Jr, in Proc. Rob. Bio. Syst., 1993, pp. 474-479.

[13] M. Otake, Y. Kagami, Y. Kuniyoshi, M. Inaba, H. Inoue, in Proc. IEEE Int. Conf. Rob. Autom., 1999, 2003, pp. 2299-2304.

[14] M. Otake, Y. Kagami, M. Inaba, H. Inoue, Robot. Auton. Syst. 2002, 40, 185-191.

[15] J. Madden, Science 2007, 318, 1094.

[16] Y. Osada, H. Okuzaki, H. Hori, Nature 1992, 355, 242-244.

[17] R. P. Hamlen, C. E. Kent, S. N. Shafer, Nature 1965, 206, 1149-1150.

[18] T. Shiga, T. Kurauchi, J. Appl. Poly. Sci. 1990, 39, 2305-2320.

[19] H. F. Schulte, in The Application Of External Power In Prosthetics And Orthotics, National Academy Of Sciences, National Research Council, Washington, DC, 1961.

[20] K. Suzumori, A. Koga, H. Riyoko, in Proc. 1994 IEEE Int. Conf. MEMS, 1994, pp. 136-141.

[21] K. Suzumori, S. Iikura, H. Tanaka, in Proc. 1991 IEEE Int. Conf. Rob. Autom., 1991, pp. 204-209. 
[22] K. Suzumori, S. Iikura, H. Tanaka, in Proc. 1991 IEEE Int. Conf. Rob. Autom., 1991, pp. 1622-1627.

[23] K. Suzumori, S. Iikura, H. Tanaka, IEEE Contr. Syst. Mag. 1992, 12, 21-27.

[24] K. Mølhave, T. Wich, A. Kortschack, P. Bøggild, Nanotechnology 2006, 17, 2434.

[25] I. Roch, P. Bidaud, D. Collard, L. Buchaillot, J. Micromech. Microeng. 2003, 13, 330.

[26] V. Seidemann, S. Bütefisch, S. Büttgenbach, Sensor. Actuat. A-Phys. 2002, 97, 457461.

[27] M. Goldfarb, N. Celanovic, Robotica 1999, 17, 181-187.

[28] J. Tedford, Robotica 1990, 8, 279-283.

[29] A. S. Brown, Mech. Eng. 2009, 131, 22-22.

[30] A. Needleman, Int. J. Solids Struct. 1977, 13, 409-421.

[31] G. M. Whitesides, "Towards a science of simplicity," can be found under http://www.ted.com/talks/george_whitesides_toward_a_science_of_simplicity.html.

[32] M. C. Boyce, E. M. Arruda, Rubber Chem. Technol. 2000, 73, 504-523.

[33] D. Nicholson, N. Nelson, Rubber Chem. Technol. 1990, 63, 368-406.

[34] According to the manufacturer's data sheet.

[35] J. McDonald, G. Whitesides, Acc. Chem. Res 2002, 35, 491-499.

[36] J. Lewis, Adv. Funct. Mater. 2006, 16, 2193-2204.

[37] M. Geissler, Y. Xia, Adv. Mater. 2004, 16, 1249-1269.

[38] M. Kubo, X. Li, C. Kim, M. Hashimoto, B. Wiley, D. Ham, G. Whitesides, Adv. Mater. 2010, 22, 2749-2752.

[39] R. K. Kramer, C. Majidi, R. J. Wood, Adv. Mater. 2010, 22, 3700-3703. 
[40] A. V. Pocius, D. A. Dillard, M. K. Chaudhury, Adhesion Science and Engineering, Elsevier, 2002. 
Figure 1: The directionality of the curvature in actuated PneuNets depends on (i) the geometry of the channels, (ii) the properties of the materials surrounding the channel, and (iii) the proximity of the channel to free surfaces. a) For channels formed in uniform materials (top row), upon pressurization, the portion that has the thinnest wall will expand first, as it requires the least amount of force (or pressure) to deform. Further pressurization continues to thin that wall and consequently bends the top surface, and forms a concave shape. When the same geometry is used to fabricate PneuNets in composite materials (bottom row of a), the stiffer material (whose Young's modulus, E, is larger) will undergo bending, and the more flexible material (lower Young's modulus) will expand. In this situation, even though the channel is closer to the surface at the bottom, it is constrained by a stiffer material, thus the bottom surface will form a concave shape. b) Finite element analysis of a channel fabricated in a homogeneous elastomer expanding under pressure. c) A series of cross-sectional slices of pressurized channels. To obtain these structures, we injected dyed Ecoflex (black) in the PneuNets, cured the dyed Ecoflex, and cut cross-sections. After filling the channels with Ecoflex, we cured and sectioned the expanded structures. Scale bar is $5 \mathrm{~mm}$.

Figure 1.

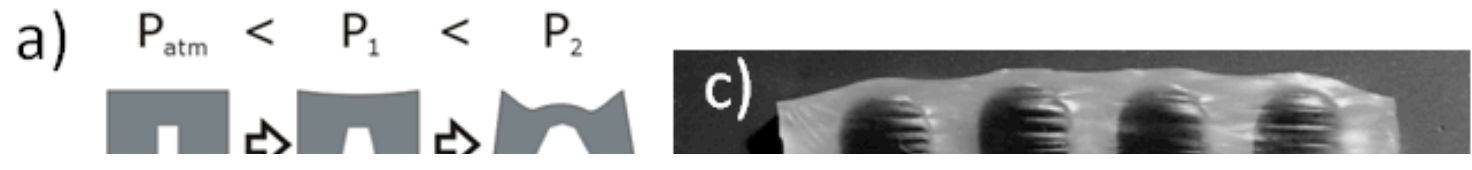


Figure 2: a) A completely elastic cycle of a PDMS-Ecoflex PneuNet undergoing pressurization and depressurization. b) Left: a fully pressurized PneuNet fabricated entirely in PDMS (any further pressurization leads to failure); right, a fully pressurized PneuNet of the same design fabricated entirely out of Ecoflex. c) With properly selected materials, it was possible to control the actuated shape of the PneuNet; three devices had channels with the same unpressurized geometries and dimensions, but had different responses to pressurization: left, the strain-limiting layer was the thickest layer of Ecoflex (bottom layer), and upon pressurization, the device formed a convex shape; middle, the strain-limiting layer was a membrane of PDMS as a top layer, and upon pressurization, the device actuated opposite of that shown to the left and formed a concave shape; right, a polyester fabric was the strain-limiting layer and the device also formed a concave shape. All structures when not pressurized were $5 \mathrm{~cm} \times 5 \mathrm{~cm} \times 5 \mathrm{~mm}$; the abbreviations for the materials are E-Ecoflex, $\mathrm{P}-\mathrm{PDMS}$, and $\mathrm{F}$ - polyester fabric.

Figure 2:

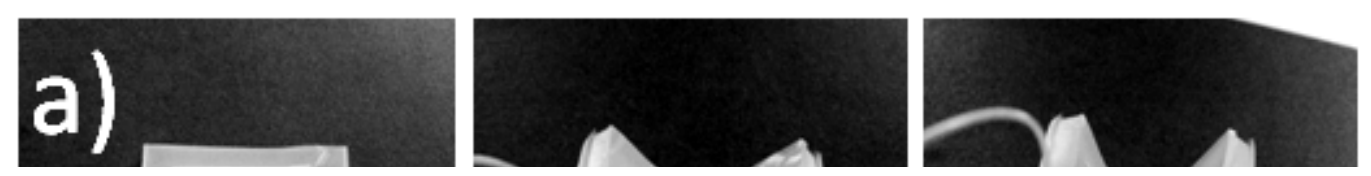


Figure 4: a) A single multifunctional device comprises multiple, independently prepared, and joined layers. The schematic shows the three-layer design of a device, which can change its curvature from concave to convex upon actuation of the two active layers pattered in Ecoflex. A PDMS membrane seals both PneuNets and also serves as a separation layer. b) The upper figure is a schematic for the geometry of the in-plane channel of the active layers in a). The tip-to-tip diameter is $9 \mathrm{~cm}$. The photographs demonstrate the fabricated device with a wide range of curvature achievable by curling upwards or downwards (from concave to convex). c) A 14-cm (tip-to-tip diameter) starfish gripper, with a modified surface facing up has thinner, but longer arms, and is capable of gripping larger, irregularly shaped objects. The top is the in-plane schematic of the distribution of channels. The arrows indicate small polyethylene tubes used to supply compressed air for actuation. 
Figure 4
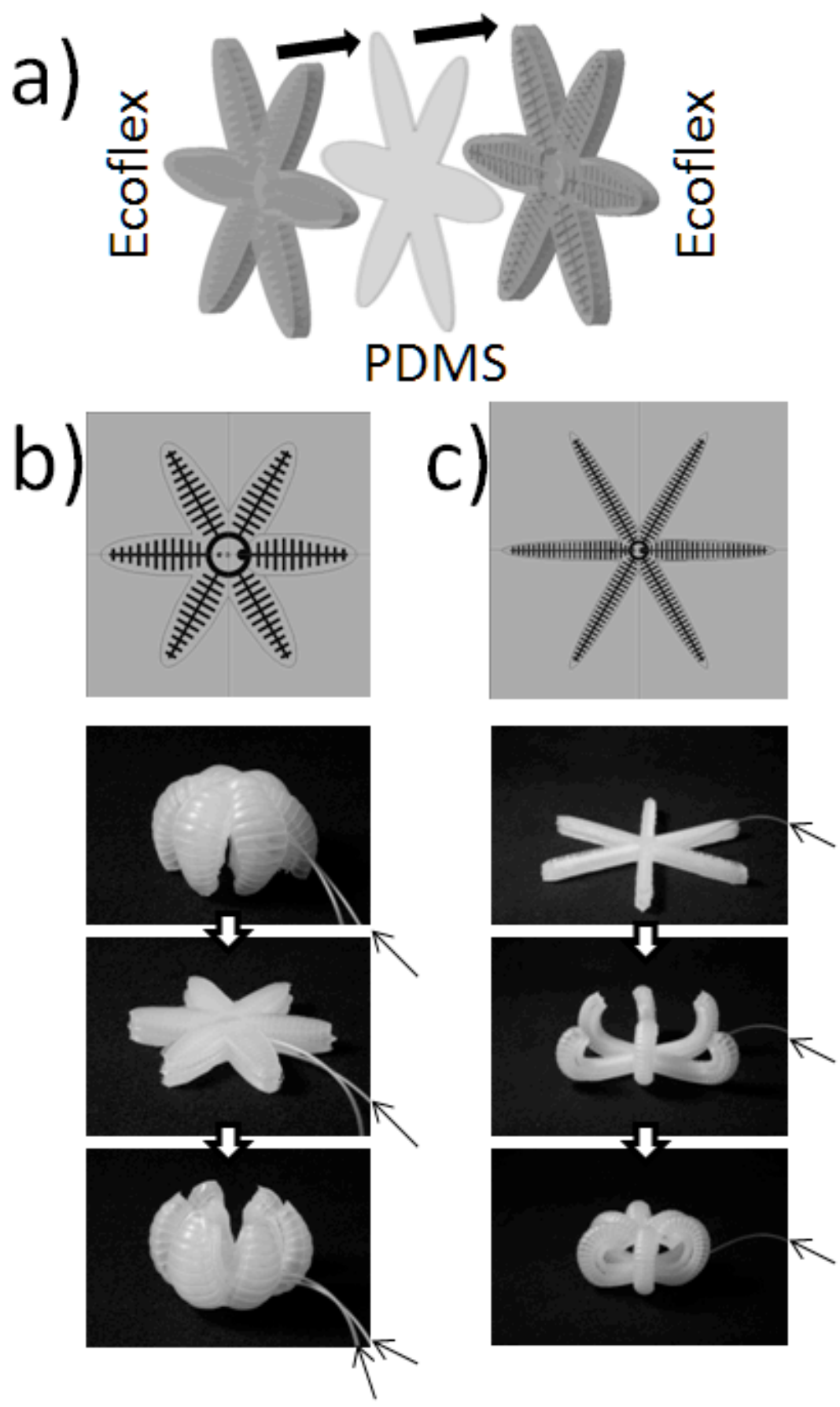
Figure 5: a) A close-up photograph showing a textured surface which has been modified independently of the PneuNets. A set of ridges formed in Ecoflex provided better traction than a flat surface (this texture is more compliant than a solid surface because half of the volume has effectively been removed, and each ridge is pliable and deforms easily). b) A photograph demonstrating an actuated leg on the gripper; the individual chambers that make up the network are visible. The minimum radius of curvature achieved in this design is less than $5 \mathrm{~mm}$.

Figure 5

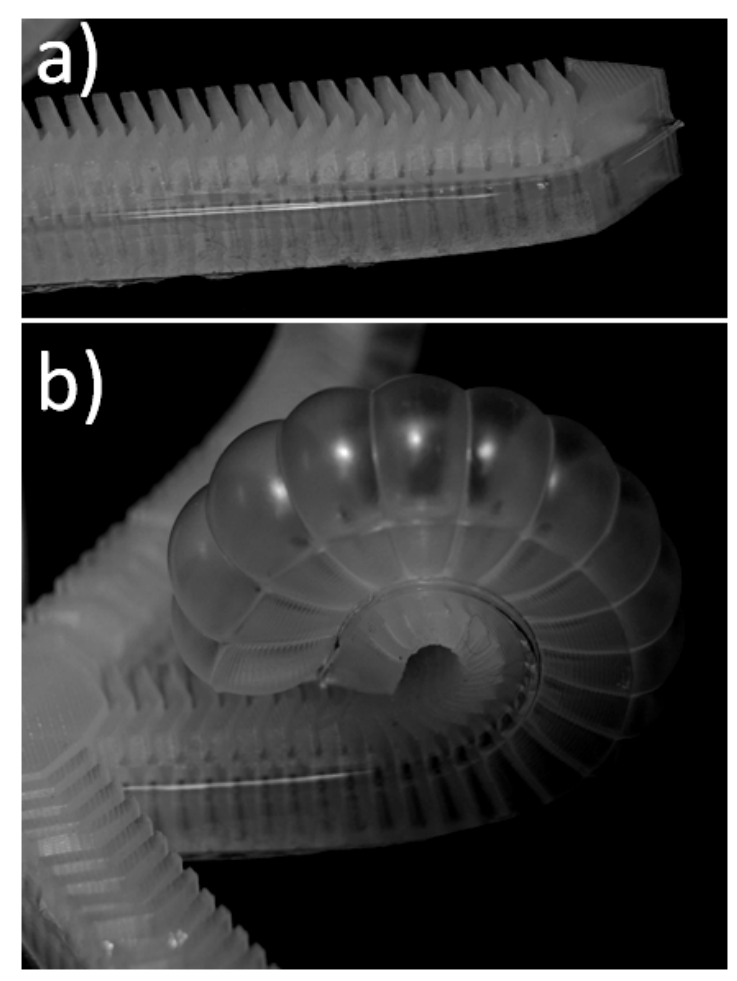


Figure 6: The left column shows a 9-cm tip-to-tip PneuNet gripping an uncooked chicken egg. A string suspends the gripper and assists in lifting the egg; a tube, visible on the left side of the gripper, runs into the central portion of the gripper to provide pressurized air for actuation. On the right, a modified version of the starfish-based gripper with 14-cm tip-to-tip distance; thinner and longer fingers can pick up a live anesthetized mouse; here also, a string (dashed arrow) suspends the gripper, and a tube (solid arrow) from the side provides compressed air. 
Figure 6
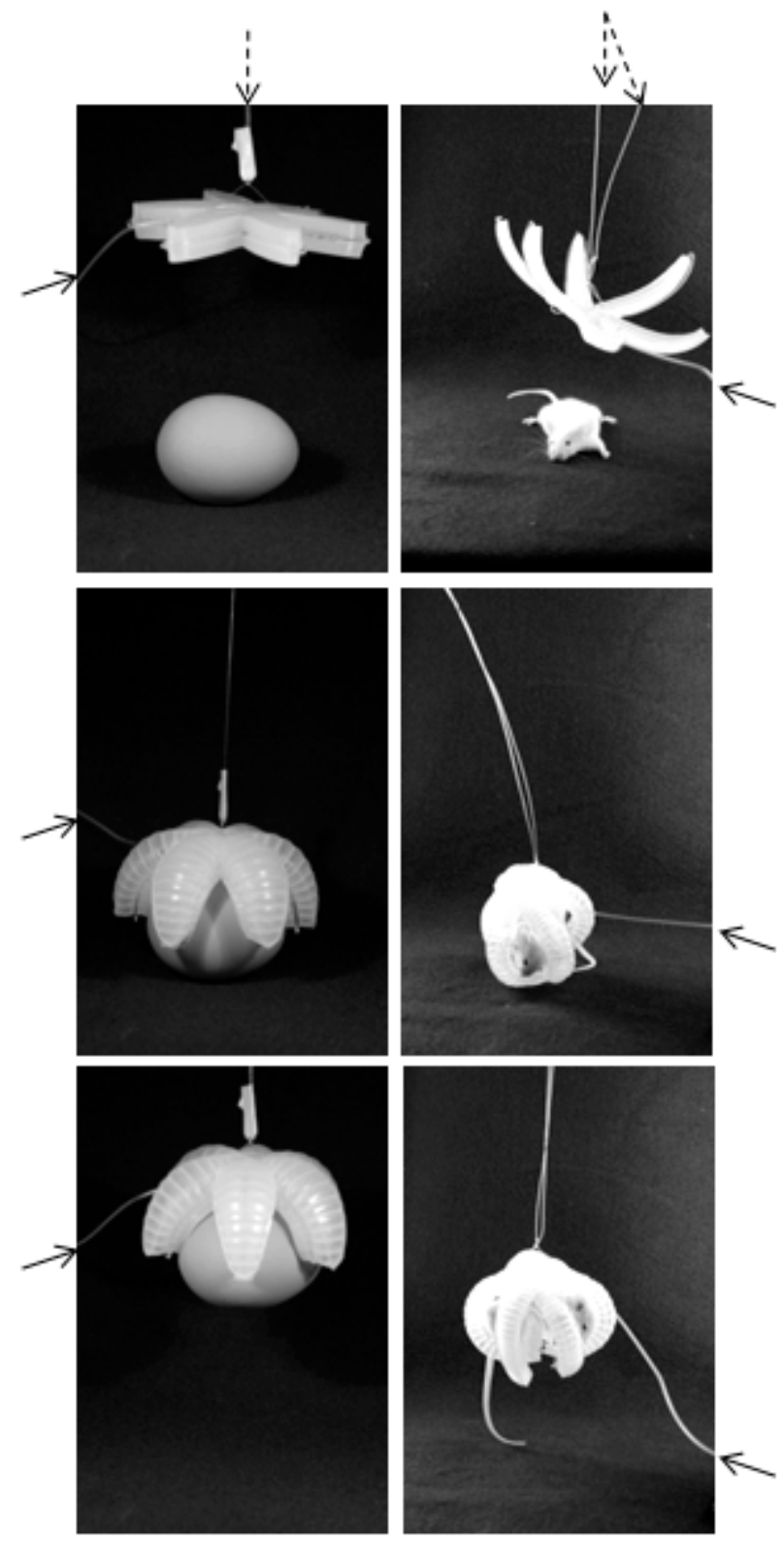